\title{
All that Clots Is Not Blood: Bone Cement Implantation Syndrome Presenting as an Intracardiac Mass and Pulmonary Embolism
}

\author{
May Anne Cheong ${ }^{10}$ Kenneth Michael Chew ${ }^{2}$ \\ ${ }^{1}$ Department of Haematology, Singapore General Hospital, \\ Singapore \\ ${ }^{2}$ Department of Cardiology, National Heart Centre, Singapore
}

Address for correspondence May Anne Cheong, Department of
Haematology, Singapore General Hospital, 20 College Road,
Singapore 169856 (e-mail: cheong.may.anne@singhealth.com.sg).

Thromb Haemost 2022;122:1432-1434.

\author{
Abstract \\ Keywords \\ - thrombosis \\ - surgery \\ - disseminated \\ intravascular \\ coagulation
}

Bone cement implantation syndrome (BCIS) is a rare and potentially fatal perioperative complication of cemented orthopaedic surgery. A computed tomography pulmonary angiogram and echocardiogram images were acquired from an 88-year-old patient who had a perioperative collapse while undergoing a revision operation for a periimplant fracture of the right femoral neck. Findings were suggestive of an intracardiac clot connected to a saddle pulmonary artery embolus. The patient also developed disseminated intravascular coagulation. Overall findings were suggestive of BCIS.
Bone cement implantation syndrome (BCIS) is a rare and potentially fatal perioperative complication of cemented orthopaedic surgery. A computed tomography (CT)-pulmonary angiogram and echocardiogram images were acquired from an 88-year-old patient with a peri-implant fracture of the right femoral neck. A new implant was inserted with bone cement. During cementation, she developed a cardiac arrest with pulseless electrical activity.

CT-pulmonary angiogram showed the intracardiac mass connected to a saddle pulmonary artery embolus. This was seen on a noncontrasted view, consistent with a radioopaque bone cement (-Fig. 1). The transthoracic echocardiogram on the apical four-chamber view showed a $2.5 \mathrm{~cm}$ pedunculated mass transiting between the right atrial and right ventricle (-Fig. 2, arrow). This mass was seen on the right ventricular outflow tract view and was connected to an echo-dense structure in the pulmonary artery (-Fig. 3, arrow). McConnell's sign was demonstrated, which is a regional pattern of right ventricular dysfunction and is an indicator of the presence of a pulmonary embolus. ${ }^{1}$ The patient also developed severe coagulopathy with a prolonged prothrombin time of 22.9 seconds, an activated partial thromboplastin time of 72.9 seconds, a decreased fibrinogen of $0.69 \mathrm{~g} / \mathrm{L}$, and an elevated D-dimer of $5.5 \mathrm{mg} / \mathrm{L}$ suggestive of disseminated intravascular coagulation.

BCIS is a rare, perioperative thrombotic phenomenon which hematologists should consider as a clinical factor and management principles defer slightly from traditional venous thromboembolism. The syndrome is characterized by hypoxia, hypotension, increased pulmonary vascular resistance, and cardiac arrest. Associated thrombotic masses have commonly been reported. ${ }^{2}$ The mechanism for thrombosis is postulated to be multifactorial, including cement embolization, complement activation, and histamine release. $^{3}$

Management of BCIS is mainly supportive based on acute critical care principles. As the contributor of cardiovascular collapse is predominately right ventricular cardiac failure, fluid resuscitation and vasopressor support should be considered. Alpha-adrenergic agents are preferred. Extracorporeal membrane oxygenation may also be considered for patients with very unstable hemodynamics. The role and efficacy of anticoagulation is not clear. ${ }^{3}$ It may also be difficult to be administered as patients often develop disseminated intravascular coagulation. Surgical or percutaneous embolectomy can be considered but usually reserved for received

November 30, 2021

accepted after revision

January 10, 2022

published online

March 7, 2022 (c) 2022. Thieme. All rights reserved. Georg Thieme Verlag KG,

Rüdigerstraße 14,

70469 Stuttgart, Germany
DOI https://doi.org/

10.1055/a-1738-1777.

ISSN 0340-6245. 


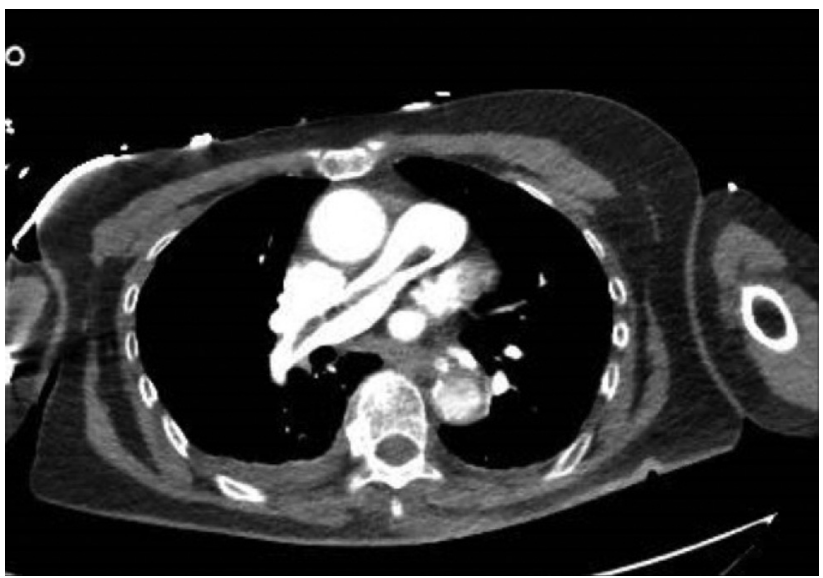

Fig. 1 CT pulmonary angiogram demonstrating saddle pulmonary embolus extending into the right ventricle and atrium. CT, computed tomography.

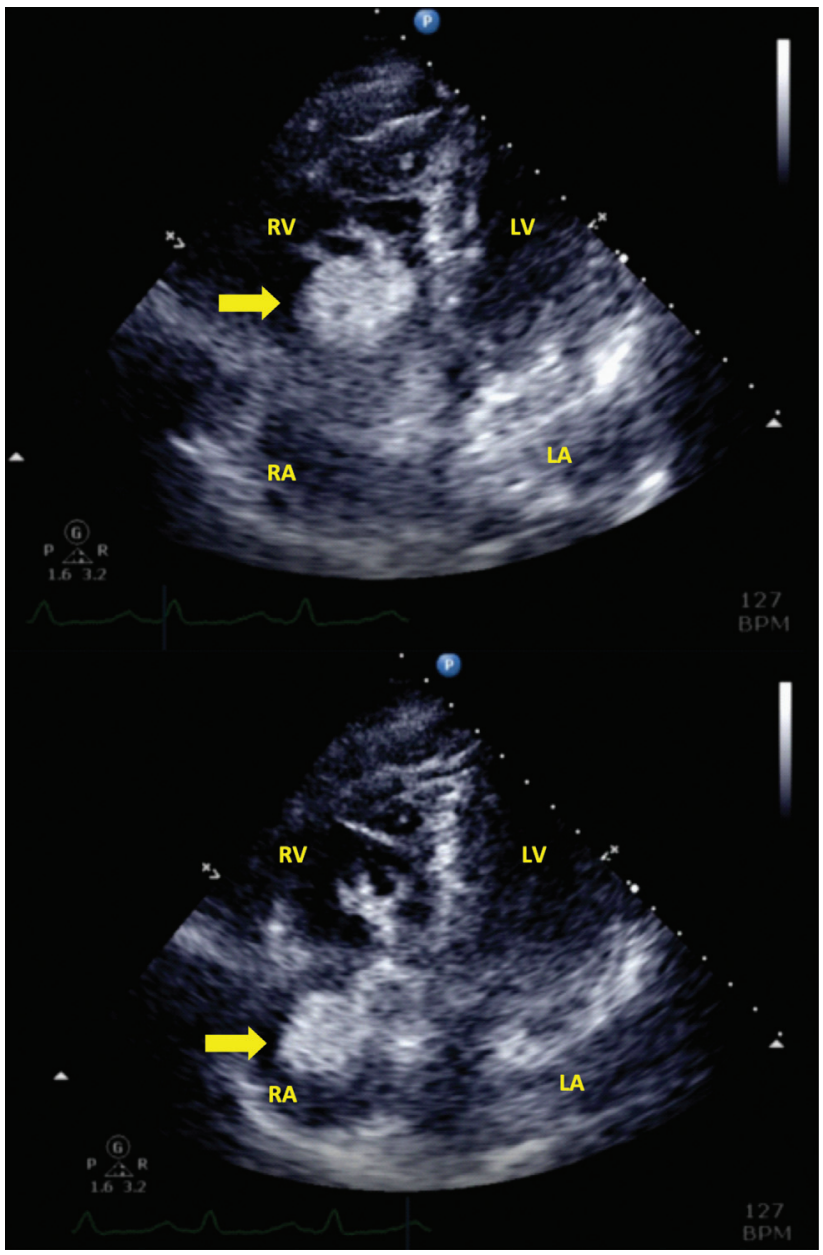

Fig. 2 Apical four-chamber view: pedunculated mass (arrow) transiting between right atrial and right ventricle. LA, left atrium; LV, left ventricle; $R A$, right atrium; RV, right ventricle.

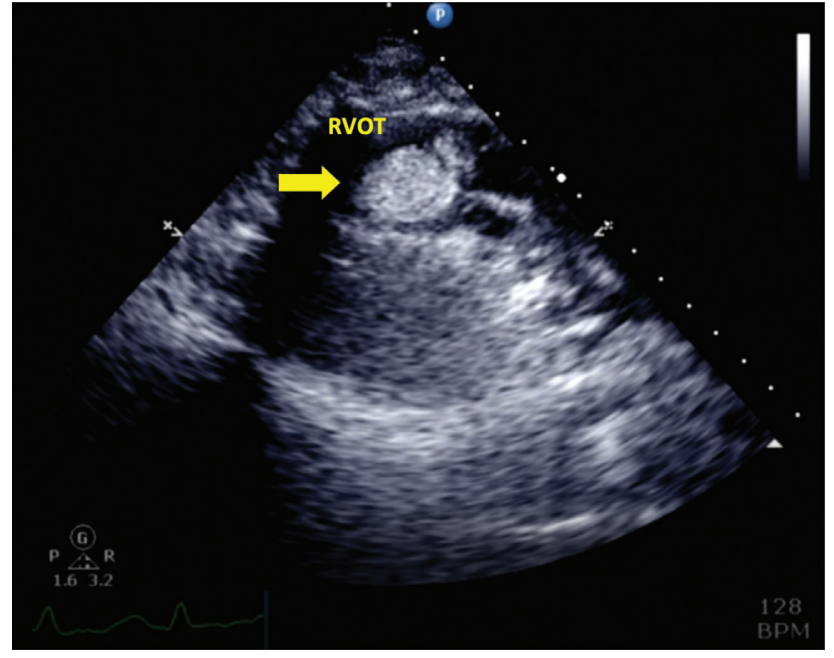

Fig. 3 Right ventricular outflow tract view: demonstrating the intracardiac mass (arrow) in the right ventricular outflow tract connected to an echo-dense structure in the pulmonary artery. RVOT, right ventricular outflow tract.

patients with central thrombus. ${ }^{4}$ The risk factors for BCIS include patient factors, e.g., old age, osteoporosis, and surgical factors, e.g., revision surgery. In light of the potentially devastating clinical consequences, for patients at high risk, preventative interventions such as increased monitoring at periods of high risk and use of cementless or low-viscosity cement prosthesis should be considered. ${ }^{3}$

The patient demised 12 hours postoperation.

\section{Author Contributions}

All authors have accepted responsibility for the entire content of this manuscript and approved its submission. M. A. Cheong and K. M. Chew wrote the manuscript.

Conflict of Interest

None declared.

\section{References}

1 Sosland RP, Gupta K. Images in cardiovascular medicine: McConnell's sign. Circulation 2008;118(15):e517-e518

2 Izumi Y, Ishihara S, Cammack I, Miyawaki I. Massive mass embolism detected by transesophageal echocardiography in bone cement implantation syndrome: a case report. JA Clin Rep 2019;5(01):5

3 Donaldson AJ, Thomson HE, Harper NJ, Kenny NW. Bone cement implantation syndrome. Br J Anaesth 2009;102(01): $12-22$

4 Wang LJ, Yang HL, Shi YX, Jiang WM, Chen L. Pulmonary cement embolism associated with percutaneous vertebroplasty or kyphoplasty: a systematic review. Orthop Surg 2012;4(03): 182-189 


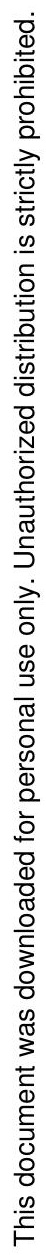

